\title{
A Spider Silk-derived Solubility Domain Inhibits Nuclear and Cytosolic Protein Aggregation in Human Cells
}

\author{
Katharina Schellhaus \\ Karolinska Institutet \\ Maria Gierisch \\ Karolinska Institutet \\ Julia Vornberger \\ Karolinska Institutet \\ Jan Johansson \\ Karolinska Institute \\ Nico Dantuma ( $\nabla$ nico.dantuma@ki.se ) \\ Karolinska Institutet
}

\section{Article}

Keywords: protein aggregation, spidroins, spider silk, solubility tag, amyloid

Posted Date: October 29th, 2021

DOI: https://doi.org/10.21203/rs.3.rs-990691/v1

License: (9) This work is licensed under a Creative Commons Attribution 4.0 International License. Read Full License

Version of Record: A version of this preprint was published at Communications Biology on May 26th, 2022. See the published version at https://doi.org/10.1038/s42003-022-03442-5. 
1 Schellhaus et al, Inhibition of protein aggregation in human cells

1 A spider silk-derived solubility domain inhibits nuclear and cytosolic protein 2 aggregation in human cells

3 Anna Katharina Schellhaus ${ }^{1}$, Maria E. Gierisch ${ }^{1}$, Julia Vornberger ${ }^{1}$, Jan Johansson ${ }^{2}$ 4 and Nico P. Dantuma ${ }^{1, *}$

5 'Department of Cell and Molecular Biology, Karolinska Institutet, Solnavägen 9, S617165 Stockholm, Sweden.

7 2Department of Biosciences and Nutrition, Karolinska Institutet, Neo, S-14183 8 Huddinge, Sweden.

9 *Correspondence: Nico P. Dantuma, Department of Cell and Molecular Biology, 10 Karolinska Institutet, Biomedicum, 7A, Solnavägen 9, S-171 65 Stockholm, Sweden; 11 Telephone: +46-8-52487384; e-mail: nico.dantuma@ki.se 
Abstract

Due to the inherent toxicity of protein aggregates, the propensity of natural, functional amyloidogenic proteins to aggregate must be tightly controlled to avoid negative consequences on cellular viability. The importance of controlled aggregation in biological processes is illustrated by spidroins, which are functional amyloidogenic proteins that form the basis for spider silk. Premature aggregation of spidroins is prevented by the $\mathbf{N}$-terminal NT domain. Here we explored the potential of the engineered, spidroin-based $\mathrm{NT}^{*}$ domain in preventing protein aggregation in the intracellular environment of human cells. We show that the $\mathrm{NT}^{*}$ domain increases the soluble pool of a reporter protein carrying a ligand-regulatable aggregation domain. Interestingly, the $\mathrm{NT}^{*}$ domain prevents the formation of aggregates independent of its position in the aggregation-prone protein. The ability of the $\mathrm{NT}^{*}$ domain to inhibit ligandregulated aggregation was evident both in the cytosolic and nuclear compartments, which are both highly relevant for human disorders linked to non-physiological protein aggregation. We conclude that the spidroin-derived $\mathrm{NT}^{*}$ domain has a generic anti-aggregation activity, independent of position or subcellular location, that is also active in human cells and propose that the NT* domain can potentially be exploited in controlling protein aggregation of disease-associated proteins.

Keywords: protein aggregation, spidroins, spider silk, solubility tag, amyloid 


\section{Introduction}

The plethora of human diseases that are linked to proteins that precipitate into insoluble aggregates highlights the importance for cells to keep their intracellular environment free of these inherently toxic protein species ${ }^{1}$. The devastating effects of aggregation-prone proteins are clearly illustrated by neurodegenerative diseases, such as Alzheimer's, Parkinson's and Huntington's disease, which are characterized by an age-dependent accumulation of insoluble protein aggregates in affected neurons or brain parenchyma ${ }^{2}$. Although neurological disorders may be overrepresented, probably due to the limited possibilities of long-lived, post-mitotic neurons to eliminate protein aggregates, protein misfolding disorders are by no means limited to neurodegeneration, but also include diseases in which other tissues are affected by aggregation-prone proteins ${ }^{3}$. Even though the exact nature of the toxic species is still subject to debate, there is a large body of evidence suggesting that the propensity of proteins to aggregate is the primary determinant for the toxicity they elicit in these diseases ${ }^{4}$. Accordingly, keeping the disease-associated proteins in a soluble state is expected to minimize their cytotoxic effects and is, as such, a desirable but challenging objective from a therapeutical perspective.

The strength, robustness and hard-to-disentangle nature of protein aggregates are at the same time unique properties that endow aggregation-prone proteins with the ability to form exceptionally rigid structures that can deal with forces that would be difficult, if not impossible, to resist by natively folded, globular proteins ${ }^{5}$. This exquisite characteristic explains the presence of amyloidogenic domains in a variety of natural proteins for which the unique features of these domains outweigh the costs of keeping the potential toxicity of these proteins at bay. A prime example is the family of amyloidogenic proteins called spidroins, which form the amyloid-like fibers that are the main constituent of spider silk, giving this biological product its unique properties and exceptional strength ${ }^{6,7}$.

Spidroins are present at very high concentrations in the spider's gland but only form safely amyloid-like fibers when secreted, which demands a stringent and tightly controlled mechanism to prevent premature aggregation in the gland. This is accomplished by the N-terminal (NT) domain of spidroins that precedes the highly repetitive amyloidogenic domain and regulates the formation of amyloid fibrils in a $\mathrm{pH}-$ and $\mathrm{CO}_{2}$-dependent fashion ${ }^{8}$. The NT domain has a dual function as it not only 
prevents premature aggregation but also promotes aggregation when appropriate by forming NT-dimers that promote oligomerization of the amyloidogenic domain ${ }^{9}$. An engineered NT domain, referred to as NT*, contains two amino acid substitutions, due to which it does not display the $\mathrm{pH}$-dependent dimerization in vitro while still preventing protein aggregation ${ }^{10}$. The $\mathrm{NT}^{*}$ domain has been explored in various experimental models for its ability to prevent aggregation of proteins-of-interest ${ }^{11-14}$. The spidrionderived $\mathrm{NT}^{*}$ anti-aggregation domain has also been found to efficiently prevent aggregation of bacterially produced recombinant proteins, underscoring its usefulness as a solubility tag ${ }^{10,12}$. Interestingly, the ability of the NT domain to prevent protein aggregation appears to be its primary, native function, unlike other commonly used solubility tags, such as glutathione $S$ transferase (GST), maltose binding protein (MBP), and ubiquitin ${ }^{15}$, which motivated us to examine its anti-aggregation potential in the natural environment where disease-associated amyloidogenic proteins have devastating effects.

In this study, we explored the anti-aggregation properties of the $\mathrm{NT}^{*}$ domain in the cytosolic and nuclear compartment of human cells using the ligand-regulatable aggregation motif $\mathrm{AgDD}^{16}$. This revealed that this spider silk-derived domain can efficiently prevent protein aggregation in cellular compartments relevant for protein misfolding diseases. This proof-of-principle study suggests that the generic antiaggregation domain of a natural spider protein may be exploitable to counteract the toxic properties of disease-associated, aggregation-prone proteins. 


\section{Results}

\section{Spider $\mathrm{NT}^{*}$ domain prevents ligand-regulatable aggregation in human cells}

Our aim was to test if the anti-aggregation properties of the $\mathrm{NT}^{*}$ domain can be exploited in preventing protein aggregation in human cells. For our experiments, we selected the ligand-regulatable aggregation domain AgDD. The AgDD domain is a modified version of a destabilization domain (DD) that targets proteins for degradation in the absence of the ligand Shield $1^{17}$. By adding an additional 10 amino-acid sequence to the $\mathrm{N}$-terminus of the DD signal, this motif has been converted to a Shield1-regulatable aggregation domain that allows rapid induction of protein aggregation by omission of the ligand ${ }^{16}$. We chose this engineered aggregation domain because of its regulatable nature and its ability to promote the formation of filamentous protein aggregates that resemble the aggregates observed with diseaseassociated proteins ${ }^{16}$. The finding that AgDD induces protein aggregation irrespective of its position in the host protein also allowed a modular approach. For easy detection and to avoid interference of protein aggregation on the folding and maturation of the fluorescent protein, we combined these domains with superfolder green fluorescent protein (sgGFP) ${ }^{18}$. Thus, the reporter proteins were composed of three modules: the AgDD aggregation domain, the $\mathrm{NT}^{*}$ anti-aggregation domain, and the superfolder green fluorescent protein (sfGFP) (Fig. 1A).

In the first set of experiments, we analyzed if the $\mathrm{NT}^{*}$ domain could reduce aggregation of a fusion protein carrying an N-terminal AgDD domain as it has been shown to induce aggregation most efficiently in this position ${ }^{16}$. The fusion proteins were transiently expressed in HeLa cells and analyzed by western blotting for protein aggregation. As expected, AgDD-sfGFP displayed Shield1-regulatable aggregation as omission of Shield1 resulted in a significant decrease in the pool of soluble AgDD-sfGFP (Fig. 1B, C). Importantly, insertion of the $\mathrm{NT}^{*}$ anti-aggregation domain in between the AgDD and sfGFP modules resulted in an increase in soluble fusion protein in the absence of Shield1 (Fig. 1B, C). Administration of Shield1 did not further increase the solubility of AgDD-NT*-sfGFP suggesting that protein aggregation was efficiently prevented by insertion of the $\mathrm{NT}^{*}$ domain.

\section{Anti-aggregation effect of $\mathrm{NT}^{*}$ is independent of position within host protein}


To test the impact of the position of the $\mathrm{NT}^{*}$ domain, we compared the ability of $\mathrm{NT}^{*}$ to prevent aggregation when placed at the amino terminus, as above, or with a centrally or carboxy terminally positioned $\mathrm{NT}^{*}$ domain (Fig. 2A). HeLa cell lines stably expressing NT*-AgDD-sfGFP, AgDD-NT*-sfGFP or AgDD-sfGFP-NT* were generated as well as a control HeLa cell line that expressed the AgDD-sfGFP reporter lacking the $\mathrm{NT}^{*}$ domain. Flow cytometry showed a homogenous expression of the transgene in each of the stable cell lines (Fig. 2B). In the AgDD-sfGFP-expressing cells, the majority of the fusion protein was detergent insoluble in the absence of Shield1 (Fig. 2C). Western blot analysis confirmed the anti-aggregation effect of the internally positioned $\mathrm{NT}^{*}$ domain in the stable cell line as no detectable signal was obtained in the detergent insoluble material with antibodies directed against GFP and $\mathrm{NT}^{*}$ even in the absence of Shield1 (Fig. 2C). This effect was indistinguishable from the effects observed with the amino and carboxy terminal $\mathrm{NT}^{*}$ domains, suggesting that its ability to prevent aggregation is not affected by the localization in the host protein (Fig. 2C). Administration of Shield1 rendered the $\mathrm{NT}^{*}$-lacking AgDD-sfGFP comparably soluble as the effect of insertion of the $\mathrm{NT}^{*}$ domain (Fig. 2D). Microscopic analysis revealed primarily cytosolic aggregates in AgDD-sfGFP-expressing cells in the absence of Shield 1 but a predominant homogenous cytosolic staining of the fusions that contained the $\mathrm{NT}^{*}$ domain, consistent with its anti-aggregation properties (Fig. 2E). Addition of Shield 1 to the culture medium did not only prevent the formation of the cytosolic AgDDsfGFP aggregates but also caused a uniform redistribution of the AgDD-sfGFP in the cytosolic and nuclear compartments (Fig. 2F). Whereas the $\mathrm{NT}^{*}$-containing fusions displayed a homogenous distribution in the absence and presence of Shield1, we observed also here a redistribution from a predominant cytosolic localization to a uniform distribution throughout the cytosolic and nuclear compartment upon administration of Shield (Fig. 2F). As this redistribution was observed for both the control AgDD-sfGFP and the NT*-containing fusions, this is likely a general effect of Shield1 on the AgDD domain and hence a feature independent of the $\mathrm{NT}^{*}$ domain.

\section{$\mathrm{NT}^{*}$ inhibits ligand-regulatable aggregation in the nucleus}

The presence of nuclear inclusions is a common phenomenon in neurodegenerative diseases ${ }^{19}$. Moreover, nuclear localization of aggregation-prone proteins has been found to be a critical for its pathologic effect in several neurodegenerative diseases ${ }^{20-}$ 
23. As the AgDD-sfGFP primarily caused cytosolic aggregates, the question remained whether $\mathrm{NT}^{*}$ can also prevent protein aggregation in the nuclear compartment. To address this question, we provided the AgDD-sfGFP and AgDD-NT*-sfGFP with a nuclear localization signal (NLS) and transiently expressed these proteins in HeLa cells. Microscopic analysis confirmed that reporter proteins efficiently localized to the nuclear compartment (Fig. 3A, B). The NLS-AgDD-sfGFP fusion precipitated in large intranuclear aggregates in the absence of Shield1 (Fig. 3A). The NT* containing fusion did not give rise to large intranuclear inclusions in the absence of Shield1 but displayed a similar, more uniform nuclear staining in the absence or presence of Shield1 (Fig. 3B). The nuclear staining with the NLS-AgDD-NT*-sfGFP had a granular appearance that did not change in appearance depending on Shield1. Western blotting confirmed that in the presence of Shield1 both reporter proteins resided in the soluble fraction (Fig. 3C, D). Withdrawal of Shield1 changed the status of the vast majority of NLSAgDD-sfGFP to detergent insoluble but this was significantly reduced by introduction of the NT* domain, which kept the majority in a detergent soluble state in the absence of Shield1 (Fig. 3C, D). A fraction of insoluble nuclear AgDD-NT*-sfGFP protein remained which was, however, largely unaffected by administration of Shield1 (Fig. 3C, D). We conclude that the $\mathrm{NT}^{*}$ domain can inhibit the Shield1-regulatable nuclear aggregation but at the same time endows the reporter protein with properties that causes a granular localization in the nuclear compartment and prevents complete solubilization. 
8 Schellhaus et al, Inhibition of protein aggregation in human cells

\section{Discussion}

Lessons learned from nature's solutions to physiological challenges can give important insights for our own attempts to modulate these processes in human diseases. The emerging understanding that proteins in an insoluble, aggregated state are not only involved in the aetiology of diseases but also of critical importance in a variety of physiological processes implies that cells must have developed their own strategies to control the potential toxicity of these amyloidogenic proteins ${ }^{5}$. Given the generic nature of protein aggregation it is maybe not surprising that a spider-derived solubility domain can prevent aggregation of human proteins ${ }^{10}$, in a similar way that it temporally and spatially controls the oligomerization of spidroins into spider silk ${ }^{9}$. In the present study, we show that this anti-aggregation property remains active in the cytosolic and nuclear compartments of human cells. This is of particular interest for the large number of human diseases that are linked to intracellular aggregation of amyloidogenic proteins.

In both the cytosolic and nuclear compartments, introduction of the NT* domain could prevent the ligand-regulatable aggregation of the reporter. This effect was independent of the position of the $\mathrm{NT}^{*}$ domain in the host protein and comparable to the solubilizing effect of the Shield1 ligand. The position-independent effect of NT* is promising when it comes to preventing aggregation of disease-associated proteins as it suggests a plasticity that may allow construction of generic tools for targeting endogenous alleles. It is noteworthy that even though the $\mathrm{NT}^{*}$ domain prevented ligand-regulatable aggregation of the nuclear targeted reporter, it at the same time gave rise to a small insoluble pool that was accompanied by a granular nuclear distribution of the reporter protein. The reason for this phenomenon is presently unclear but appears to be a feature of the $\mathrm{NT}^{*}$ domain itself.

Long-term efforts have focused on the identification of small molecules and peptides that can prevent, inhibit or reverse protein aggregation ${ }^{24}$. Although there is little doubt that such drug-like molecules would be preferable from a therapeutic perspective, exploiting inhibition of protein aggregation by natural domains has some advantages that justify efforts to better understand their mode of action and applicability in the context of human diseases. Importantly, whereas compounds that modulate protein aggregation are unlikely to be specific for disease-associated proteins and may interfere at the same time with physiological processes driven by amyloidogenic conversion of proteins, the cis-acting nature of $\mathrm{NT}^{*}$ and other natural anti-aggregation 
9 Schellhaus et al, Inhibition of protein aggregation in human cells

211 domains will confine their effect to the host protein only while leaving other processes

212 unaffected. This rather unique feature may be hard to accomplish with drug-like 213 molecules but become a more realistic endeavor with increased understanding of 214 natural domains that have evolved to control protein aggregation. With this in mind, we 215 anticipate that our finding of the conserved anti-aggregation activity of a spider protein 216 in human cells may stimulate further interest in these domains. 
10 Schellhaus et al, Inhibition of protein aggregation in human cells

\section{Materials \& Methods}

\section{Constructs}

The AgDD-sfGFP construct was purchased from Addgene (\#78289). All PCR amplifications were done according to the manufacturer's instructions for Phusion HF DNA polymerase (New England Biolabs, Ipswich, USA). The DD domain was PCR amplified using the primers 5'-CAG TGC TGG GAA TTC AAA TTC GGC CAC CAT GCT GG-3' (fwd) and 5'-AAG TTC TTC TCC TTT GCT GAA TTC TTC CGG TTT TAG AAG CTC CAC-3' (rev). The NT*-tag was PCR amplified using the previously described $\mathrm{NT}^{*}$-A $\beta 42$ construct $^{12}$ and the primers 5'-GTC TCA TCA TTT TGG CAA AGA TGT CAC ACA CTA CAC CAT G-3' (fwd) and 5'-CAT GGT GGC CGA ATT TGA ATT CCC AGC ACT GAC-3' (rev). The PCR products were purified from a $1 \%$ agarose gel using the Qiagen gel extraction kit (Qiagen, Venlo, Netherlands) and inserted in EcoR1 digested AgDD-sfGFP using NEbuilder HiFi DNA Assembly Master Mix (New England Biolabs, Ipswich, USA). In order to clone AgDD-NT*-sfGFP, a similar procedure was performed with the primers 5'-AAA CCG GAA GAA TTC ATG TCA CAC ACT ACA CCA TG-3' (fwd) and 5'-AGT TCT TCT CCT TTG CTG AAG CTA GCT GAA TTC CCA GC-3' (rev). For the DD domain, the primers 5'-GTC TCA TCA TTT TGG CAA AGA ATT CGC CAC CAT GCT GG-3' (fwd) and 5'-TAG TGT GTG ACA TGA ATT CTT CCG GTT TTA GAA GCT CCA C-3' (rev) were used. The AgDD-sfGFP-NT* was originally constructed in the EGFP-N1 vector (Clontech Laboratories, Mountain View, USA) by digesting EGFP-N1 with the restriction enzymes BamHI and Notl. The AgDD-sfGFP was PCR amplified using the primers 5'-CGA CGG TAC CGC GGG CCC GGG ATC CAT GCT GGC CCT GAA GCT G-3' (fwd) and 5'-TAG TGT GTG ACA TGA ATT CGG ATC CTT TGT AGA GCT CAT CCA TG-3' (rev). NT* was amplified using the primers 5'-TGA GCT CTA CAA AGG ATC CGA ATT CAT GTC ACA CAC TAC ACC-3' (fwd) and 5'-TGA TTA TGA TCT AGA GTC GCG GCC GCT TAT GAA TTC CCA GCA CTG AC-3' (rev). The AgDD-sfGFP and NT* PCR products were inserted in the EGFP-N1 backbone according to the procedure outlined above giving rise to AgDDsfGFP-NT* (EGFP-N1). The AgDD-sfGFP-NT* open reading frame was PCR amplified from AgDD-sfGFP-NT* (EGFP-N1) the using the primers 5'-AAA CAT TCT TGG ACA CAA ACT CGA GTA CAA CTT TAA CTC ACA C-3' (fwd) and 5'-TAA GCT GCA ATA AAC AAG TTA ACT TAT GAA TTC CCA GCA CTG-3' (rev). The PCR 
product was used to swap the insert in AgDD-sfGFP plasmid, which was excising with the restriction enzymes $\mathrm{Hpal}$ and Xhol, with AgDD-sfGFP-NT* using the procedure outlined above. NLS-AgDD-sfGFP was received from Add-gene (\#80625). The NLSAgDD-NT*-sfGFP was generated by PCR amplifying the open reading frame from NLS-AgDD-sfGFP (Addgene \#80625) using the primers 5'-GTC TCA TCA TTT TGG CAA AGA ATT CGC CAC CAT GCC AC-3' (fwd) and 5'-TAG TGT GTG ACA TGAA TTC TTC CGG TTT TAG AAG CTC CAC-3' (rev). The PCR product was inserted with the PCR amplified NT*-tag using NEbuilder HiFi DNA Assembly Master Mix.

\section{Generation of stable cell lines}

Parental HeLa cells were transfected with either AgDD-sfGFP, NT*-AGDD-sfGFP, AgDD-NT*-sfGFP or AgDD-sfGFP-NT* together with 0,2 $\mu$ g PiggyBac Transposase Expression Vector using Lipofectamine 3000 (Life Technologies, Carlsbad, USA) according to manufacturer's instructions. Transfected cells were successively sorted by flow cytometry for sfGFP expression at 3 and 10 days after transfection using the ARIA III FACS sorter followed by expansion of the sorted cells.

\section{Fluorescence microscopy}

Stable cell lines were seeded on coverslips in the presence or absence of $1 \mu \mathrm{M}$ Shield1 (Takara Bio, Kyoto, Japan). Cells were fixed with 4\% paraformaldehyde (PFA) in phosphate buffered saline (PBS) for $15 \mathrm{~min}$ at 24 or $48 \mathrm{hrs}$ postseeding. Coverslips were washed, stained with $2 \mu \mathrm{g} / \mathrm{ml}$ Hoechst 33342 (Thermo Fischer Scientific) in PBS for $15 \mathrm{~min}$ and mounted with homemade Mowiol/DABCO. Coverslips were imaged using a Zeiss LSM 880 confocal microscope with a Plan-Apo 40x/1.3 Oil objective (Zeiss, Jena, Germany) or a Zeiss LSM 710 confocal microscope equipped with a CApochromat 40X/1.2 water objective.

\section{Soluble and insoluble fractionation}

HeLa cells stably expressing AgDD-sfGFP, NT*-AgDD-sfGFP, AgDD-NT*-sfGFP or AgDD-sfGFP-NT* ${ }^{*}$ were incubated in the absence or presence of $1 \mu \mathrm{M}$ Shield-1 for 16 - 
24 hrs. Cells were washed twice with PBS, harvested in lysis buffer A (150 mM NaCl, $50 \mathrm{mM}$ Tris $\mathrm{pH}$ 7.5, $0.5 \mathrm{mM}$ EDTA, 1\% Triton X-100, 1x complete protease inhibitor cocktail (Roche, Basel, Switzerland), $10 \mu \mathrm{M}$ MG132) and incubated on ice for $50 \mathrm{~min}$. A small part of the sample was taken and mixed with 0.5 of its volume of $4 x$ reducing NUPAGE LDS sample buffer (Invitrogen, Carlsbad, USA) and boiled ("Input”). The remaining sample was spun down at $21.130 \mathrm{~g}$ and $4{ }^{\circ} \mathrm{C}$ for $30 \mathrm{~min}$. The supernatant was mixed with 0.5 of its volume of $4 x$ reducing NuPAGE LDS sample and boiled ("Soluble fraction"). The pellet was washed twice with lysis buffer and resuspended in equal volumes of lysis buffer and $4 x$ reducing NuPAGE LDS sample buffer. These samples were boiled and sonicated ("Insoluble fraction"). Samples were run on 4-12\% NuPAGE Bis-Tris gels (Invitrogen, Carlsbad, USA) with MOPS running buffer and transferred to PVDF membranes. The membranes were blocked in $5 \%$ milk dissolved in Tris-buffered saline (TBS) $+0,1 \%$ Tween and incubated first with primary antibodies against GFP (Abcam, AB290, rabbit polyclonal, 1:2500 in milk or Roche, \#1814460, mouse, 1:5000 in milk), against the $\mathrm{NT}^{*}-\operatorname{tag}^{25}$ (rabbit, 1:1500 in milk) and GAPDH (Abcam, ab29485, rabbit, 1:2500), followed by IRDye 800CW goat anti-mouse IgG and IRDye 680RD goat anti-rabbit IgG secondary antibodies (both 1:5000) (LI-COR, Lincoln, USA) and imaged with the Odyssey infrared imaging system (LI-COR, Lincoln, USA). The intensity of the GFP bands in the "Soluble fraction" and "Insoluble fraction" from three independent experiments of the soluble and insoluble fractions were quantified using ImageJ.

\section{Acknowledgements}

We thank the members of the Dantuma lab, Dr. Henrik Biverstål, Dr. Per Nilsson and Dr. Makoto Shimozawa (Department of Neurobiology, Care Sciences and Society, Karolinska Institutet) for helpful input. This work was supported by the Swedish Research Council (N.P.D. 2016-02479, J.J. 2020-02434), the Swedish Cancer Society (N.P.D. CAN 2018/693), CIMED (J.J.) and StratNeuro (N.P.D., J.J.). A.K.S. and M.E.G. were supported by research fellowships from the Deutsche Forschungsgemeinschaft (DFG) (SHE 2079/1-1; GZ:GI-1329/1-1).

\section{Author contributions}


13 Schellhaus et al, Inhibition of protein aggregation in human cells

312 A.K.S. performed all experiments except for the flow cytometry; M.G. performed the 313 flow cytometry experiment; A.K.S, J.V. performed the microscopy experiment shown 314 in Fig. 2 and 3; J.J., N.P.D. wrote the manuscript; J.J., N.P.D. coordinated the project; 315 all authors edited and approved the final manuscript. 


\section{Figure legends}

Figure 1. Spider $\mathrm{NT}^{*}$ domain prevents ligand-regulatable aggregation in human cells

A) Schematic representation of the fusion protein AgDD-sfGFP and AgDD-NT*-sfGFP.

B) Hela cells were transiently transfected with AgDD-sfGFP or AgDD-NT*-sfGFP, incubated in the absence or presence of $1 \mu \mathrm{M}$ Shield-1 and fractionated into Tx100 soluble (S) and Tx100 insoluble/SDS soluble (P) fractions. (I) shows the whole cell lysate/input before fractionation. Western blots were done with antibodies against GFP and GAPDH.

C) Quantification of the GFP band of the relative distribution of the soluble and insoluble fractions.

Figure 2. Anti-aggregation effect of $\mathrm{NT}^{*}$ is independent of position within host protein

A) Schematic representation of the fusion protein with the $\mathrm{NT}^{*}$ domain in different positions.

B) Hela cells were stably transfected with the fusion proteins depicted in (A) and analysed by flow cytometry.

C-D) Hela cells stably expressing AgDD-sfGFP, NT*-AgDD-sfGFP, AgDD-NT*-sfGFP or AgDD-sfGFP-NT* were incubated for $16-24 \mathrm{hrs}$ in the absence (C) or presence of 1 $\mu \mathrm{M}$ Shield-1 (D) and fractionated into Tx100 soluble (S) and Tx100 insoluble/SDS soluble $(\mathrm{P})$ fractions. Input of whole cell lysate before fractionation are shown (I). Western blots were performed with antibodies directed against GFP, the NT*-tag and GAPDH. Quantifications of the GFP band of the relative distribution of the soluble and insoluble fractions from three independent experiments are shown

E-F) Hela cells stably expressing AgDD-sfGFP, NT*-AgDD-sfGFP, AgDD-NT*-sfGFP or AgDD-sfGFP-NT* in the absence $(E)$ or presence $(F)$ of $1 \mu \mathrm{M}$ Shield-1 were fixed in 4\% PFA and counterstained with Hoechst. Scale bar $20 \mu \mathrm{m}$. 
Figure 3. NT* inhibits ligand-regulatable aggregation in the nucleus

346 A-B) Hela cells were transiently transfected with NLS-AgDD-sfGFP or NLS-AgDD$347 \mathrm{NT}^{*}$-sfGFP and incubated in the absence $(\mathbf{A})$ or presence $(\mathbf{B})$ of $1 \mu \mathrm{M}$ Shield-1. Cells 348 were fixed $24 \mathrm{hrs}$ after transfection with 4\% PFA and counterstained with Hoechst. 349 Scale bar $20 \mu \mathrm{m}$.

350 C) Hela cells transiently transfected with NLS-AgDD-sfGFP or NLS-AgDD-NT*-sfGFP 351 were incubated for 16-24 hrs in the absence of presence of $1 \mu \mathrm{M}$ Shield-1 and 352 fractionated into Tx100 soluble (S) and Tx100 insoluble/SDS soluble (P) fractions. 353 Input of whole cell lysate before fractionation are shown (I) Western blots were 354 performed with antibodies against GFP, the NT*-tag and GAPDH.

355 D) Quantifications of the GFP band of the relative distribution of the soluble and 356 insoluble fractions from three independent experiments are shown. 


\section{References}

1) Sherman, M. Y. \& Goldberg, A. L.Cellular defenses against unfolded proteins: a cell biologist thinks about neurodegenerative diseases. Neuron. 29, 15-32., (2001).

2) Ciechanover, A. \& Brundin, P.The ubiquitin proteasome system in neuordegenerative diseases: sometimes the chicken, sometimes the egg. Neuron. 40, 427-446, (2003).

3) Carrell, R. W. \& Lomas, D. A.Conformational disease. Lancet. 350, 134-138., (1997).

4) Bucciantini, M. et al.Inherent toxicity of aggregates implies a common mechanism for protein misfolding diseases. Nature (London). 416, 507-511., (2002).

5) Chuang, E., Hori, A. M., Hesketh, C. D. \& Shorter, J.Amyloid assembly and disassembly. J Cell Sci. 131, (2018).

6) Johansson, J. \& Rising, A.Doing What Spiders Cannot-A Road Map to Supreme Artificial Silk Fibers. ACS Nano. 15, 1952-1959, (2021).

7) Rising, A. \& Johansson, J.Toward spinning artificial spider silk. Nat Chem Biol. 11, 309-315, (2015).

8) Andersson, M. et al.Carbonic anhydrase generates $\mathrm{CO} 2$ and $\mathrm{H}+$ that drive spider silk formation via opposite effects on the terminal domains. PLoS Biol. 12, e1001921, (2014).

9) Kronqvist, N. et al.Sequential pH-driven dimerization and stabilization of the $\mathrm{N}$-terminal domain enables rapid spider silk formation. Nat. Commun. 5, 3254, (2014).

10) Kronqvist, N. et al.Efficient protein production inspired by how spiders make silk. Nat Commun. 8 , 15504, (2017).

11) Abdelkader, E. H. \& Otting, G.NT*-HRV3CP: An optimized construct of human rhinovirus $143 C$ protease for high-yield expression and fast affinity-tag cleavage. J Biotechnol. 325, 145-151, (2021).

12) Abelein, A. et al.High-yield Production of Amyloid-beta Peptide Enabled by a Customized Spider Silk Domain. Sci Rep. 10, 235, (2020).

13) Sarr, M. et al.A spidroin-derived solubility tag enables controlled aggregation of a designed amyloid protein. FEBS J. 285, 1873-1885, (2018).

14) Schmuck, B. et al.Expression of the human molecular chaperone domain Bri2 BRICHOS on a gram per liter scale with an E. coli fed-batch culture. Microb Cell Fact. 20, 150, (2021).

15) Ki, M. R. \& Pack, S. P.Fusion tags to enhance heterologous protein expression. Appl Microbiol Biotechnol. 104, 2411-2425, (2020).

16) Miyazaki, Y. et al.A method to rapidly create protein aggregates in living cells. Nat. Commun. 7, 11689, (2016).

17) Banaszynski, L. A., Chen, L. C., Maynard-Smith, L. A., Ooi, A. G. \& Wandless, T. J.A rapid, reversible, and tunable method to regulate protein function in living cells using synthetic small molecules. Cell. 126, 995-1004, (2006).

18) Pedelacq, J. D., Cabantous, S., Tran, T., Terwilliger, T. C. \& Waldo, G. S.Engineering and characterization of a superfolder green fluorescent protein. Nat Biotechnol. 24, 79-88, (2006).

19) Ross, C. A. \& Poirier, M. A.Protein aggregation and neurodegenerative disease. Nat. Med. 10 Suppl, S10-17, (2004).

20) Bichelmeier, U. et al. Nuclear localization of ataxin-3 is required for the manifestation of symptoms in SCA3: in vivo evidence. J Neurosci. 27, 7418-7428, (2007).

21) Klement, I. A. et al.Ataxin-1 nuclear localization and aggregation: role in polyglutamine-induced disease in SCA1 transgenic mice. Cell. 95, 41-53, (1998).

22) Saudou, F., Finkbeiner, S., Devys, D. \& Greenberg, M. E.Huntingtin acts in the nucleus to induce apoptosis but death does not correlate with the formation of intranuclear inclusions. Cell. 95, 55-66., (1998).

23) Sowa, A. S. et al.Karyopherin alpha-3 is a key protein in the pathogenesis of spinocerebellar ataxia type 3 controlling the nuclear localization of ataxin-3. Proc Natl Acad Sci U S A. 115, E2624E2633, (2018).

24) Herbst, M. \& Wanker, E. E.Therapeutic approaches to polyglutamine diseases: combating protein misfolding and aggregation. Curr Pharm Des. 12, 2543-2555, (2006). 
17 Schellhaus et al, Inhibition of protein aggregation in human cells

409

25) Andersson, M., Holm, L., Ridderstrale, Y., Johansson, J. \& Rising, A.Morphology and composition of the spider major ampullate gland and dragline silk. Biomacromolecules. 14, 2945-2952, (2013).

411 


\section{Figure 1}

A

\begin{tabular}{|l|l|}
\hline AgDD & sfGFP \\
\hline
\end{tabular}

\begin{tabular}{|l|l|l|}
\hline AgDD & NT* & sfGFP \\
\hline
\end{tabular}

B

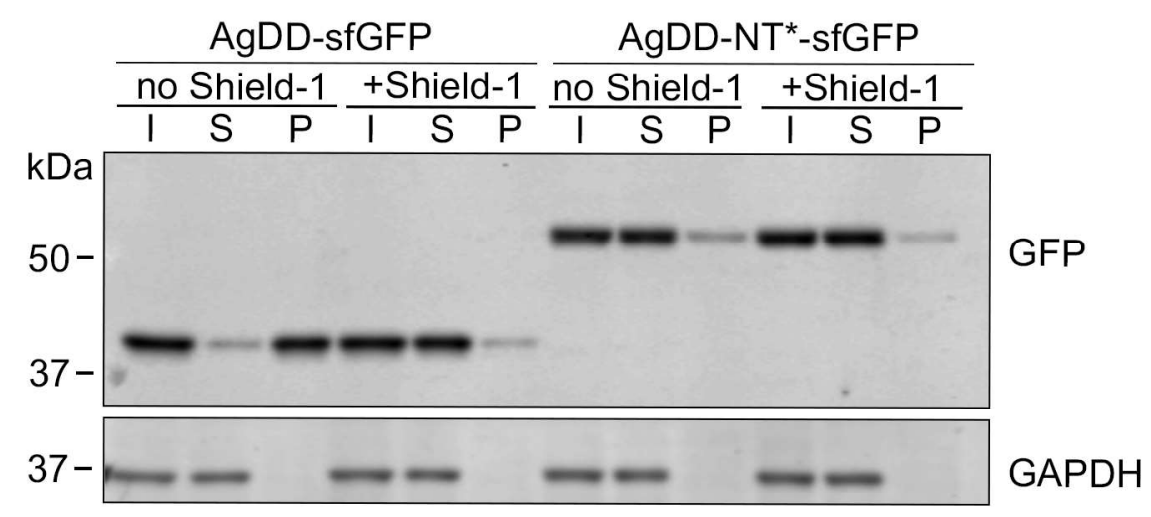

C

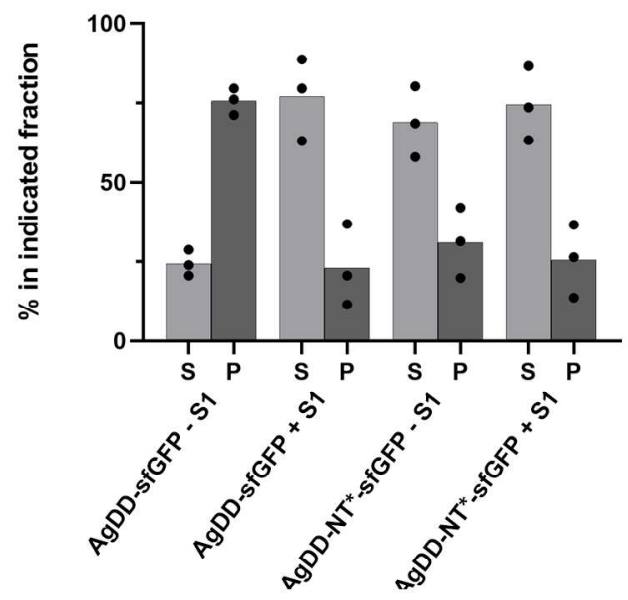


A

\begin{tabular}{|l|l|l|}
\hline AgDD & NT* $^{*}$ & sfGFP \\
\hline
\end{tabular}

\begin{tabular}{|l|l|l|}
\hline NT* $^{*}$ & AgDD & sfGFP \\
\hline
\end{tabular}

\begin{tabular}{|l|l|l|}
\hline AgDD & sfGFP & NT* $^{*}$ \\
\hline
\end{tabular}

C

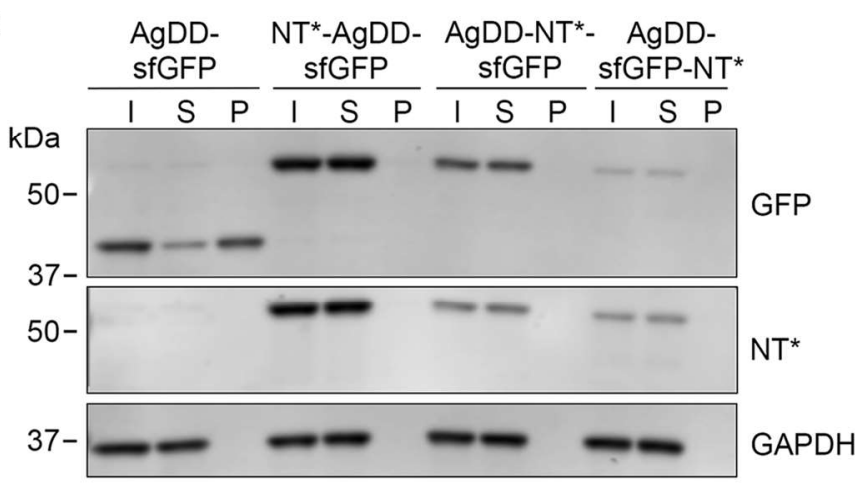

D

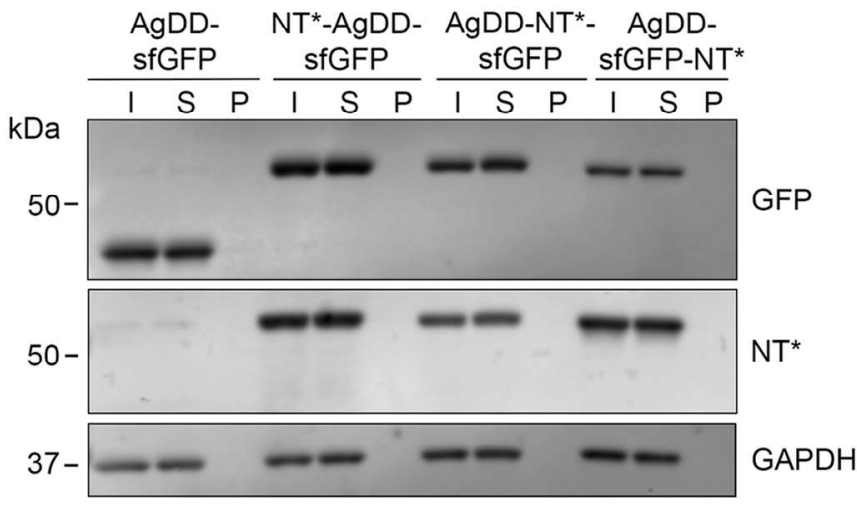

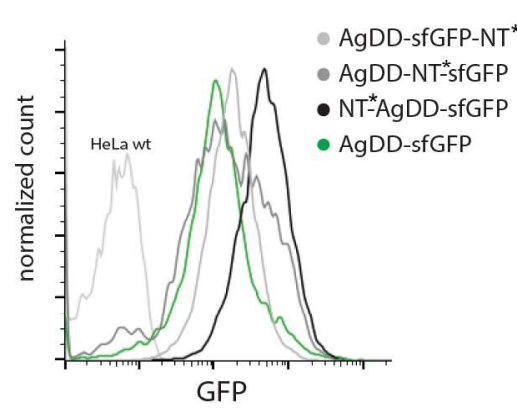

no Shield-1
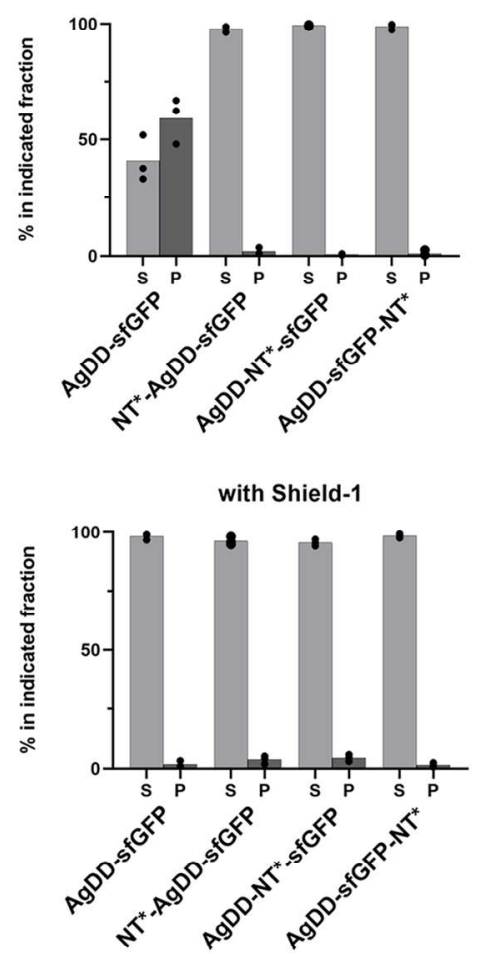

E

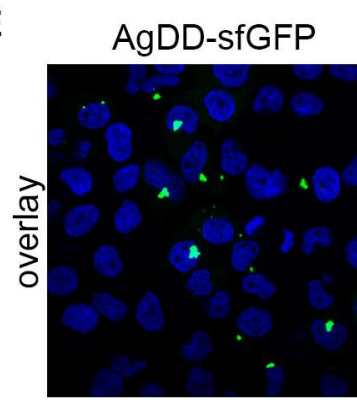
NT*-AgDD-sfGFP

AgDD-NT*-sfGFP

AgDD-sfGFP-NT*
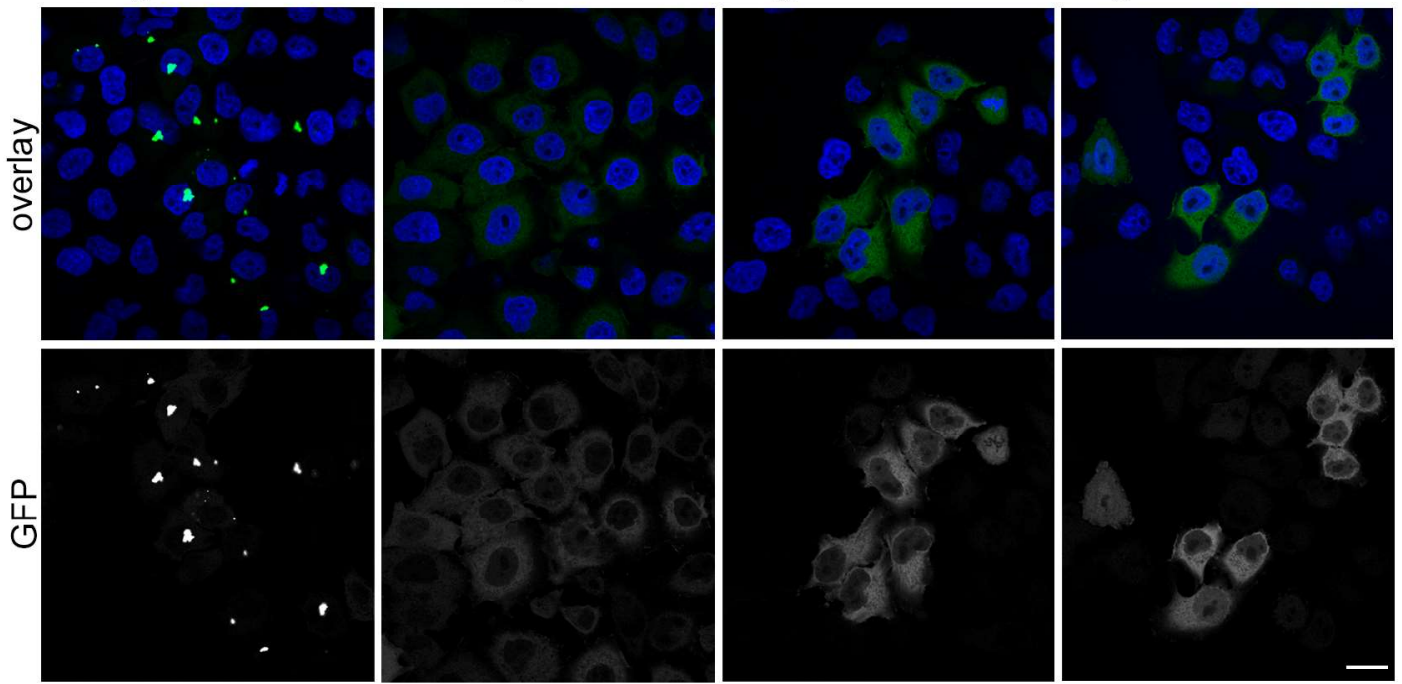

F

AgDD-sfGFP

NT*-AgDD-sfGFP

AgDD-NT*-sfGFP

AgDD-sfGFP-NT*
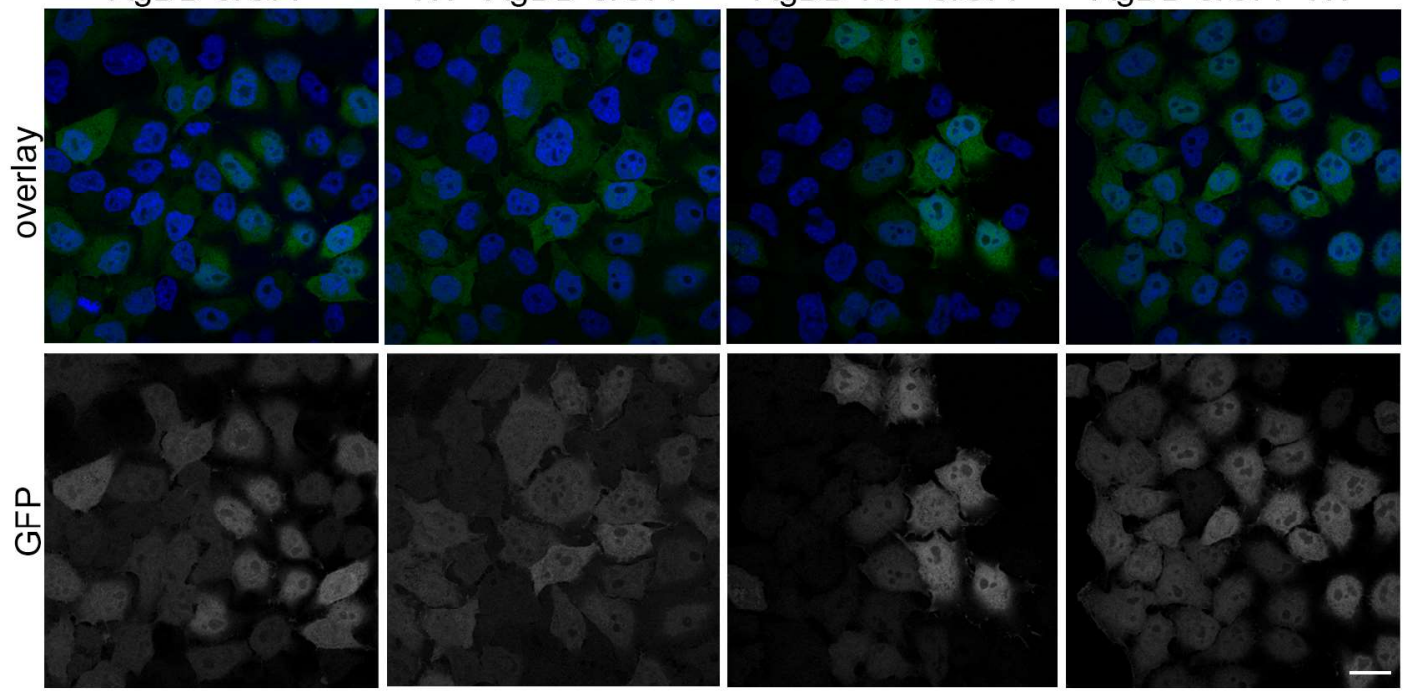
Figure 3

A

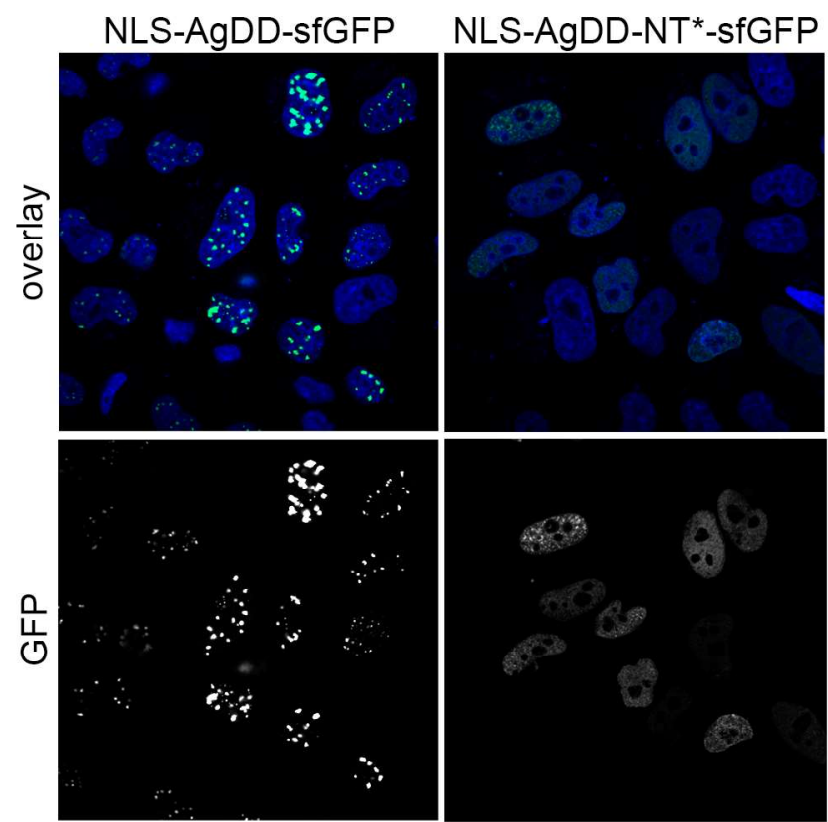

C

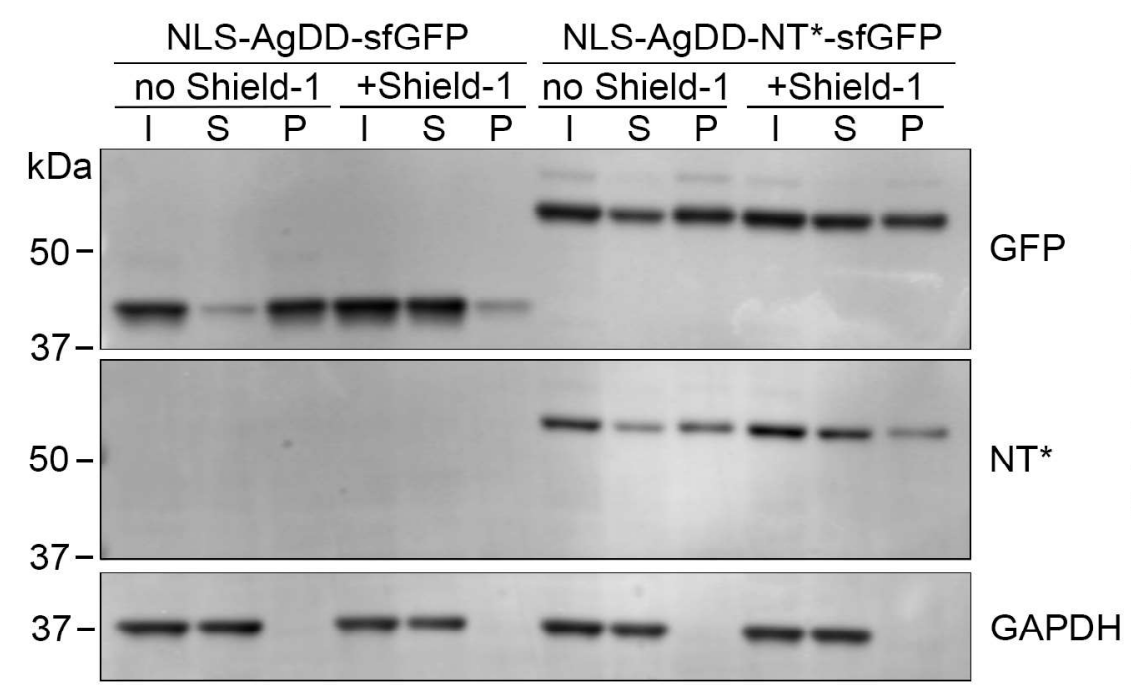

B

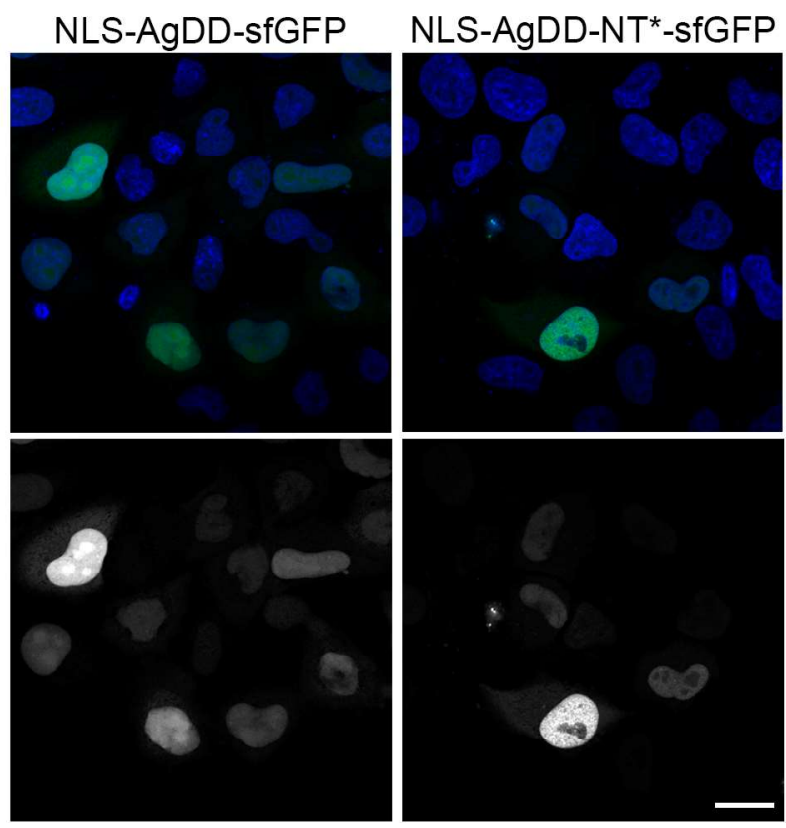

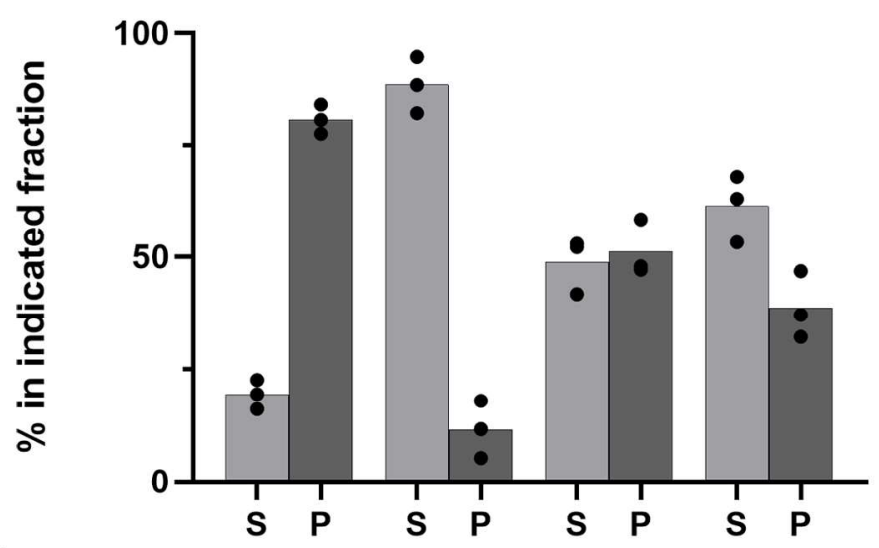

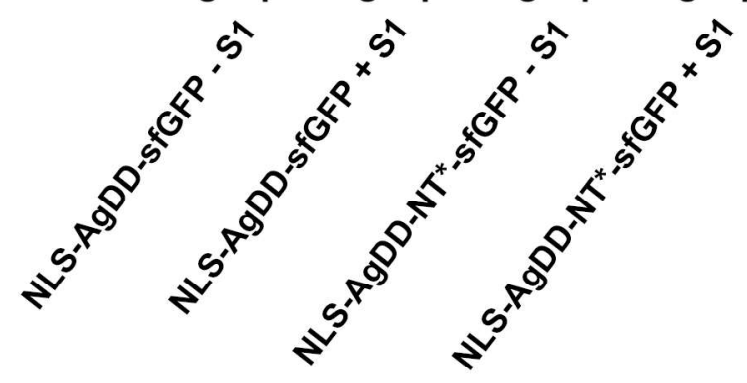

